\title{
Phase transitions in Pareto optimal complex networks
}

\author{
Luís F. Seoane ${ }^{1,2}$ and Ricard Solé ${ }^{1,2,3}$ \\ ${ }^{1}$ ICREA-Complex Systems Lab, Universitat Pompeu Fabra-PRBB, Dr. Aiguader 88, 08003 Barcelona, Spain \\ 2 Institut de Biologia Evolutiva, UPF-CSIC, Passg Barceloneta, 08003 Barcelona \\ 3 Santa Fe Institute, 1399 Hyde Park Road, New Mexico 87501, USA
}

\begin{abstract}
The organization of interactions in complex systems can be described by networks connecting different units. These graphs are useful representations of the local and global complexity of the underlying systems. The origin of their topological structure can be diverse, resulting from different mechanisms including multiplicative processes and optimization. In spatial networks or in graphs where cost constraints are at work, as it occurs in a plethora of situations from power grids to the wiring of neurons in the brain, optimization plays an important part in shaping their organization. In this paper we study network designs resulting from a Pareto optimization process, where different simultaneous constraints are the targets of selection. We analyze three variations on a problem finding phase transitions of different kinds. Distinct phases are associated to different arrangements of the connections; but the need of drastic topological changes does not determine the presence, nor the nature of the phase transitions encountered. Instead, the functions under optimization do play a determinant role. This reinforces the view that phase transitions do not arise from intrinsic properties of a system alone, but from the interplay of that system with its external constraints.
\end{abstract}

PACS numbers: 64.60.aq, 64.60.Bd, 64.60.-i, 87.55.de

\section{INTRODUCTION}

Optimization is a key goal in engineered systems and is traditionally assumed to be part of the intrinsic dynamics of natural evolving systems [1. The engineering perspective, associated to man-made objects and structures, is specially obvious when dealing with large-scale, interconnected units, as it occurs in very large integrated circuit design $[2-4]$ or spatially-extended infrastructures such as power grids [5? -7] and transportation or distribution networks [6 12. In these cases, engineers cope with interfering constraints related to materials, space, packing, wiring, or dissipation costs. The staggering complexity of these designed systems can be addressed by algorithms that deal with multidimensional problems.

In biological systems, important network topologies have been shown to result from optimality 13,14 . These include transportation networks in living organisms [15[19] where optimization is reached by means of fractal trees that guarantee a low cost and efficient location of resources. Similarly, neural circuits display optimal features over a wide range of scales [4, 20 24]. The packing and interconnectivity in some cortical areas seem compatible with design principles shared by high-density electronic designs 4 .

In all the previous examples tradeoffs between efficiency and cost are present. Packing many components in a given spatial domain is desirable because of cost minimization of connections, but dissipation of energy or wiring constraints will also be at work. What kinds of topologies result when considering multiple constraints? This problem has been addressed by explicitly introducing efficiency measures $E$ (such as average path length) along with cost constraints $C$ (such as number of connections of a given graph) 25-27]. A similar example in another field models languages as a network of associations between objects and words, and considers language evolution through a least effort process 28 30. Here, the cost-efficiency conflict is mapped onto coding/decoding efforts for users of an economic (while ambiguous) language. Both in the linguistic and the network examples, the tension between opposite demands leads to phase transition phenomena. We can wonder, in a more general note, when and how will phase transitions arise disregarding of the details of the problem in hand.

Within the context of network optimization, we consider the set $\Gamma$ of all connected networks $\gamma \in \Gamma$ involving $N$ nodes and any number of links. Latter on we will define an efficiency $(E(\gamma))$ and a cost $(C(\gamma))$ based on the structure of each network $\gamma$, so that a series of optimization problems can be posed. For each such graph, we can also introduce a global energy function $\Omega(\gamma)$ that takes into account our optimization goals. The most straightforward way to do this is through a linear combination:

$$
\Omega(\gamma, \lambda)=\lambda E(\gamma)+(1-\lambda) C(\gamma),
$$

with $\lambda \in[0,1]$ a tunable parameter that weights the impact of each contribution. This is precisely the strategy in previous accounts of these problems 25 31. If $\lambda=1$ only efficiency constraints will be at work, whereas $\lambda=0$ would ignore this component.

Such a global energy function results in very illustrative visualizations of the optimization process through the notion of a potential landscape. Assuming minimization, more optimal network architectures lay deeper in a potential well when we plot $\Omega(\gamma, \lambda)$ for every $\gamma$ superimposed on an arbitrary network morphospace, as in Fig. 1(a). Note, however, that this is a limited picture: a fixed value of $\lambda$ is necessary to generate one potential landscape. Changing the parameter modifies the landscape rendered by $\Omega(\gamma, \lambda)$ and, accordingly, the underlaying optimization problem. To achieve a more general compre- 
hension we should not only allow scenarios with different values of $\lambda$, but we must also question the hypothesis of linearity introduced by equation (1). Therefore, we consider Pareto (or Multi Objective) Optimization [32 34, whose solution is not a global optimizer dependent on external parameters (i.e. the absolute minimum of some potential landscape), but a collection of solutions that attempt to satisfy both (cost and efficiency) constraints simultaneously. These Pareto optimal designs constitute the so-called Pareto front: the most optimal tradeoff possible between the targets involved (Fig. 1(c)).

In this paper we look at such tradeoffs for a series of optimization problems defined upon complex networks. How the different constraints are satisfied depending on a series of factors is interesting in itself; but if we relate the Pareto optimal designs to the linear optimization problem posed by equation (1), a richer phenomenology unfolds. This stems from a deep connection between the Pareto front and phase transitions and other important features of thermodynamic theory [35. In this context, the energy landscapes that we can generate through equation (1) often result very useful to complete the picture.

The paper is organized as follows. Sec. III provides the basic formalism of Pareto optimization and the connection between Pareto optimality and statistical mechanics [35. This theory homes in phase transitions for the problems investigated and is the framework used to analyze most of the results obtained. Also in Sec. II we pose a series of Multi Objective Optimizations upon complex networks whose solutions are detailed in Sec. III and discussed, with closing remarks, in Sec. IV Appendix A explains some numerical aspects of the current work.

\section{PHASE TRANSITIONS IN THE PARETO FORMALISM}

A recent contribution proposed a multiobjective optimization approach to statistical mechanics that generalizes key concepts in thermodynamics for any Pareto optimality problem [35. The fundamental connection is between the Pareto front and the Gibbs surface. This surface (defined through the thermodynamic potential $G=G(U, S, V))$ is linked to the equilibrium state of a thermodynamic species. Then, its concavities and nondifferentiable edges underly the existence of first and second order phase transitions [36, 37.

A similar point can be made for systems that optimize a set $T_{f}$ of target functions $T_{f}=\left\{t_{1}, \ldots, t_{K}\right\}$, whose Pareto front encodes phase transitions and critical points in its shape. This connection between Pareto optimal systems and thermodynamics is explained at length in [35. There, general Pareto optimal designs (not necessarily networks) that minimize an arbitrary number $K \geq 2$ of targets is discussed. Here we sketch the theory with just $K=2$ optimization targets and using connected networks with fixed number of nodes $(\gamma \in \Gamma)$.
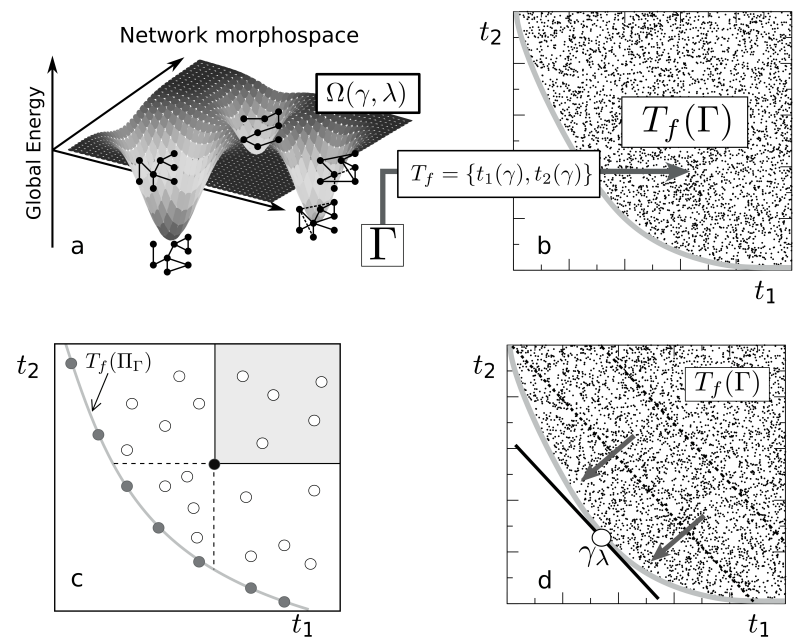

FIG. 1: A two dimensional example of Pareto optimality. (a) $\gamma \in \Gamma$ are all possible connected networks with a given number of nodes. They populate some network morphospace where we seek those graphs minimizing some measurable feature. If we deal with just one fitness function, an energy landscape can be defined and the optima are easily found at the bottom of energy wells. (b) If more than one optimization target are at play, this landscape picture falls apart and we need to adopt a Pareto optimization approach. Then our task is to find a set of Pareto optimal solutions $\left(\Pi_{\Gamma} \subset \Gamma\right)$ that minimizes all targets (here $t_{1}$ and $t_{2}$ ) simultaneously. These functions map each network $\gamma \in \Gamma$ into $\mathbb{R}^{2}$. The subset of Pareto optimal solutions is mapped into the Pareto front (thick gray curve). Along this curve it is not possible to improve both $t_{1}$ and $t_{2}$ at the same time. (c) It is convenient to introduce Pareto dominance. The black circle represents a net with lower $t_{1}$ and $t_{2}$ than (thus it Pareto dominates) those within the gray square. That same filled circle is dominated by those networks projected between the dashed lines and the Pareto front. (d) Recovering a single objective optimization through a linear combination of the targets (Eq. (1)) is akin to choosing a direction in $\mathbb{R}^{2}$ and seeking the extreme of the front along that direction.

This suffices to illustrate the relevant aspects of the theoretical framework. We remit the reader to 35 for further details.

Henceforth, $T_{f}=\left\{t_{1}, t_{2}\right\}$ are any two real valued functions that we can measure on any network. These functions project each network into $T_{f}(\gamma)=\left(t_{1}(\gamma), t_{2}(\gamma)\right)$, a point in the plane that we term target space (Fig. 1(a)(b)). In that plane we can solve the Multi Objective Optimization (MOO) problem consisting of the simultaneous minimization of both targets. Therefore we define Pareto dominance (Fig. 1(c)): A network $\gamma_{x} \in \Gamma$ dominates another $\gamma_{y} \in \Gamma$ (and we note it $\gamma_{x} \prec \gamma_{y}$ ) if

$$
\begin{array}{r}
t_{k}\left(\gamma_{x}\right) \leq t_{k}\left(\gamma_{y}\right) \forall k=1,2 ; \\
\exists k^{\prime} \in\{1,2\} \quad \mid \quad t_{k^{\prime}}\left(\gamma_{x}\right)<t_{k^{\prime}}\left(\gamma_{y}\right) .
\end{array}
$$

In this case $\gamma_{x}$ is objectively better than $\gamma_{y}$ and we can dismiss the later. It is often the case that pairs of net- 
works are mutually non dominated. Then we cannot choose between them without introducing a bias towards either $t_{1}$ or $t_{2}$. We have to avoid this bias to find Pareto optimal designs. We say that a network $\gamma_{x} \in \Gamma$ is Pareto optimal if it does not exist any other $\gamma_{y} \in \Gamma$ such that $\gamma_{y} \prec \gamma_{x}$. Hence

$$
\Pi_{\Gamma}=\left\{\gamma_{x} \in \Gamma \mid \nexists \gamma_{y} \in \Gamma, \gamma_{y} \prec \gamma_{x}\right\}
$$

is the set of all Pareto optimal solutions, which constitutes the solution of the MOO problem.

The set $\Pi_{\Gamma} \subset \Gamma$ is projected onto the target plane through $T_{f}\left(\Pi_{\Gamma}\right)$ where it represents a limiting frontier of the whole $T_{f}(\Gamma)$, known as the Pareto front. For $K=2$ this frontier is a curve that implements a bijective function of $t_{1}$ and $t_{2}$ (Fig. 1(c)). $\Pi_{\Gamma}$ is not a standard global optimizer. Instead, it comprises a collection of valid networks that embody the optimal tradeoff between many targets such that, as we move through it, we cannot improve $t_{1}$ without worsening $t_{2}$ and vice-versa.

We can now add a further demand that a global energy function

$$
\Omega(\gamma ; \lambda)=\lambda t_{1}(\gamma)+(1-\lambda) t_{2}(\gamma)
$$

be minimized. (This equation is equivalent to Eq. 1 with the more general $t_{1,2}(\gamma)$ instead of efficiency and cost. We rewrite it here for convenience.) Note that this is the simplest Single Objective Optimization (SOO) that we can built with $T_{f}$. Indeed, each possible $\lambda \in[0,1]$ poses a different SOO problem, potentially with a distinct global solution. We check now how solutions of this SOO family are related.

By setting fix a value of $\lambda$ we choose a direction in the $t_{1}-t_{2}$ plane along which the minimization proceeds (Fig. 11(d)). From Eq. 4. networks projected onto a straight line with slope $d=-\lambda /(1-\lambda)$ have the same global energy $\Omega$. Networks on straight lines pushed further against the front present lower energies, until one solution $\gamma_{\lambda} \in \Pi_{\Gamma}$ is singled out as global minimum of $\Omega(\lambda)$. It usually lays where the slope of the front is precisely $d=-\lambda /(1-\lambda)$. If the front $\Pi_{\Gamma}$ is convex and its derivatives are well defined, we can smoothly sample the front by slowly tuning $\lambda$. Any appropriate measurement that we perform on $\gamma_{\lambda}$ will be a smooth, differentiable function of $\lambda$ itself.

If a cavity exists in $T_{f}\left(\Pi_{\Gamma}\right)$, the global solutions bypass those that lay inside (Fig. 2(a)). For a certain value $\lambda^{c}$, two very different solutions $\left(\gamma_{\lambda^{c}}^{1}\right.$ and $\left.\gamma_{\lambda^{c}}^{2}\right)$ located far apart in the $t_{1}-t_{2}$ plane are simultaneously optimal. Solutions between $\gamma_{\lambda^{c}}^{1}$ and $\gamma_{\lambda^{c}}^{2}$ (inside the cavity) never get to be global optima. For $\lambda<\lambda^{c}$ we remain at one side of the cavity where a smooth sample of the Pareto front is still possible. The same happens for $\lambda>\lambda^{c}$ at the other side of the cavity. But at the characteristic value $\lambda^{c}$ there is a sudden jump between $\gamma_{\lambda^{c}}^{1}$ and $\gamma_{\lambda^{c}}^{2}$ and, consequently, in any order parameter that we can measure on $\gamma_{\lambda}$ when plotted as a function of $\lambda$ (Fig. 2(b)). This is a first order phase transition. A similar transition happens if
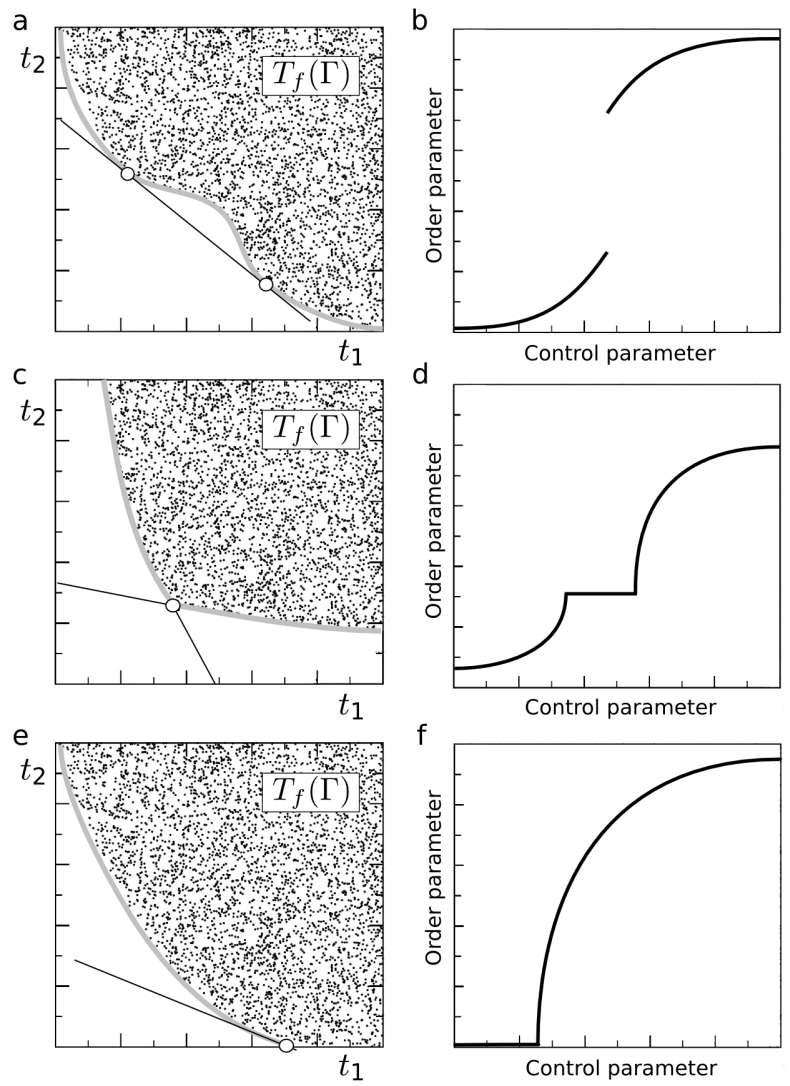

FIG. 2: Phase transitions in Pareto optimal systems. (a) A first order phase transition takes place in MOOs whose Pareto front presents a cavity. Solutions of the convex hull of the front are SOO global optima for a range of $\lambda$, while those inside the cavity are not. (b) This renders a gap in any adequate, measurable property of the global solutions. (c) Second order phase transition are associated to ill-defined derivatives - i.e. sharp edges in the front. Those designs at the edge are persistently optimal foe a range of the control parameter $\lambda$. (d) Hence, any property of the global solution is constant as a function of that same control parameter for the designated interval. (e) Second order phase transitions might also take place at the extremes of the Pareto front. We say then that the front has an abrupt ending (or that it ends in an abrupt manner) as in the lower-right extreme of this front. (f) Any order parameter presents the well-known functional dependence of second order phase transitions.

the Pareto front is a straight line (then $\lambda^{c}=-d^{c} /\left(1-\lambda^{c}\right)$, where $d^{c}$ is the slope of the front) with the singularity that all Pareto optimal solutions are also global optima for $\lambda=\lambda^{c}$.

If the front is convex but its slope is ill-defined anywhere (Fig. 2(c)) there is a range of values $\lambda \in\left(\lambda^{-}, \lambda^{+}\right)$ for which the global optimum remains the same. Because the optimum does not change within this singular range, if we plot any order parameter its derivative (with respect to $\lambda$ ) will be zero. The same derivative will be different from zero anywhere else. This results in a characteristic plot for any order parameter (Fig. 2(d)) associated to 
second order phase transitions.

Such non-differentiability of the front might be shifted all the way towards one of its extremes. For $\lambda \in(0,1)$ we seek global optima where the slope of the front is any $-\lambda /(1-\lambda) \Rightarrow d \in(-\infty, 0)$. Well-behaved Pareto fronts should roll smoothly with slopes that completely span the $d \in(-\infty, 0)$ interval; otherwise the front will be exhausted while more SOO problems can still be defined. If these slope values are not exhausted we say that the Pareto front ends in an abrupt manner, meaning that its slope as we approach its leftmost end is some $d_{-}^{*}>-\infty$, or its slope as we approach its rightmost end is some other $d_{+}^{*}<0$. These abrupt endings of the front will be optima already for some $\lambda^{*}=-d_{ \pm}^{*} /\left(1-d_{ \pm}^{*}\right)$. We can still define SOO problems for $\lambda$ beyond these limits, for which the solutions at the corresponding extremes of the front will be persistently optimal (Fig. 2(e)). This implies again a discontinuity in the derivative of any order parameter, the fingerprint of second order transitions (Fig. 2(f)).

\section{A. Multiobjective optimization of complex networks}

Complex graphs are a great testbed to illustrate this theoretical framework. They allow us to define problems of increasing difficulty where first and second order transitions arise. Seminal work on network optimization addressed the problem from an SOO perspective [11, 25, 26, 31, 38, so some of our results can be put in context. Another advantage of complex networks is that good optimizers can be produced in the computer, simplifying the empirical work.

We propose three problems based on the conflict between the average path length between nodes and the density of edges, which roughly inform us about diffusion efficiency [39] (for which low average path length is desired) and implementation costs (lower for sparser networks). Consider first the topological (or standard) average path length:

$$
\langle l\rangle^{t}(\gamma)=\frac{1}{Z_{\langle l\rangle}^{t}} \sum_{i, j} \frac{d_{i j}^{t}(\gamma)}{2},
$$

where $d_{i j}^{t}(\gamma)$ denotes the distance (in number of edges) between nodes $n_{i}, n_{j} \in \gamma$ along the shortest path that connects them; and the topological (or standard) link density:

$$
\rho^{t}(\gamma)=\frac{1}{Z_{\rho}^{t}} \sum_{i, j} \frac{a_{i j}(\gamma)}{2},
$$

where the adjacency matrix $A(\gamma)=\left\{a_{i j}(\gamma)\right\}$ presents $a_{i j}(\gamma)=1$ if two nodes are linked in $\gamma$ and $a_{i j}(\gamma)=$ 0 otherwise. $Z_{\langle l\rangle}^{t}$ and $Z_{\rho}^{t}$ are normalization constants discussed below.

The superindices in $\langle l\rangle^{t}(\gamma), \rho^{t}(\gamma)$ indicate that we deal with the topological (or standard) average path length and link density, in which edges cost 1 unit. Geometric costs can be included if nodes are distributed, e.g., over a Euclidean space. Let $d_{i j}^{g}(\gamma)$ be the Euclidean length of the shortest path connecting $n_{i}$ and $n_{j}$ in network $\gamma$ - i.e. the sum of the Euclidean lengths of the edges in the shortest path between these nodes provided that $\gamma$ is embedded in some geometric space. We introduce the geometric (or weighted) average path length:

$$
\langle l\rangle^{g}(\gamma)=\frac{1}{Z_{\langle l\rangle}^{g}} \sum_{i, j} \frac{d_{i j}^{g}(\gamma)}{2} .
$$

The shortest Euclidean distance possible between two nodes $l_{i j}(\gamma)$ only enters equation $(7)$ if a direct link between $n_{i}$ and $n_{j}$ is present in $\gamma$ (in that case $d_{i j}^{g}(\gamma)=$ $\left.l_{i j}(\gamma)\right)$. This $l_{i j}(\gamma)$ allows us to introduce the geometric (or weighted) link density:

$$
\rho^{g}(\gamma)=\frac{1}{Z_{\rho}^{g}} \sum_{i, j} \frac{a_{i j}(\gamma) l_{i j}(\gamma)}{2} .
$$

Just as before, $\langle l\rangle^{g}(\gamma)$ and $\rho^{g}(\gamma)$ (note the superindexes indicating their geometric dependence) are normalized by $Z_{\langle l\rangle}^{g}$ and $Z_{\rho}^{g}$. A clique, or fully connected network $\left(\gamma_{C}\right)$, has the shortest average path length possible always. As we will see later, this means that $\gamma_{C}$ is Pareto optimal always, so we base our normalization on it: $Z_{\langle l\rangle}^{t / g}=\sum_{i, j} d^{g}\left(i, j ; \gamma_{C}\right) / 2, Z_{\rho}^{t}=\sum_{i, j} a_{i j}\left(\gamma_{C}\right) / 2$, and $Z_{\rho}^{g}=\sum_{i, j} a_{i j}\left(\gamma_{C}\right) l\left(i, j ; \gamma_{C}\right) / 2$.

We combine $\langle l\rangle^{t / g}(\gamma)$ and $\rho^{t / g}(\gamma)$ as targets in different ways to generate three MOO problems:

(A) Fully topological problem, with $t_{1}=\langle l\rangle^{t}(\gamma)$ and $t_{2}=\rho^{t}(\gamma)$. Note that the geometry does not play any role in this case.

This version was originally studied in [25, 26] from an SOO perspective. From that approach, only the clique and star graphs appear relevant (as discussed in [26]) as the representatives of two phases at either side of a discontinuous phase transition. We show how this fits parsimoniously within the framework presented in 35. But besides, we discuss now the whole Pareto front - its relevant shape and some of its constituents. This front includes nontrivial complexities well differentiated from the star and clique, and it presents connections to critical systems discussed elsewhere [40].

(B) Partly geometrical problem, with $t_{1}=\langle l\rangle^{t}(\gamma)$ (the same as above) and $t_{2}=\rho^{g}(\gamma)$. Geometry, through $t_{2}$, plays a relevant role now. Since the disposition of the nodes in space matters, we study this MOO in two different cases: i) nodes scattered randomly over the $[0,1] \times[0,1]$ square in $\mathbb{R}^{2}$ and ii) nodes spaced evenly over a circle of radius 1 .

In this problem we still use the topological average path length, meaning that we seek to minimize the 
number of hops or the number of relay stations between arbitrary pairs of nodes. To think about this problem we can picture an infrastructure such as a subway network whose contractor wishes to minimize the length of line built while the users want to avoid transfers between lines.

(C) Fully geometrical problem, with $t_{2}=\langle l\rangle^{g}(\gamma)$ and $t_{2}=\rho^{g}(\gamma)$. In this case the geometrical cost is important for all targets involved. Again, the disposition of the nodes matters and again we study: i) nodes scattered randomly over the $[0,1] \times[0,1]$ square in $\mathbb{R}^{2}$ and ii) nodes spaced evenly over a circle of radius 1 .

\section{RESULTS}

Three relevant topologies indicate major feats of all our Pareto fronts. The most prominent one is the clique: a fully connected network that presents the largest number of links possible, thus maximizes edge density and minimizes the average path length always. This guarantees that the clique is always Pareto optimal. It marks the top-left boundary of the Pareto front, as illustrated in Fig. 3(a). This is true for all problems considered in this paper.

The star presents a hub to which all other nodes are connected, while non-hubs are not connected to each other. There are $N$ possible star graphs. If geometry is not considered, all of them are equivalent. When geometry intervenes and nodes are spaced over a circle all $N$ stars are equivalent as well. All possible trees consist of as many edges as the star but, if geometry matters, only the minimum spanning tree (MST) minimizes always the edge density $\left(t_{2}\right)$. The MST is Pareto optimal whenever geometry is relevant and it always indicates the end of the Pareto front opposite to the clique (at its bottom-right).

\section{A. Fully topological problem}

This case has been studied as an SOO through equation (4) 25, 26]. That solution is incomplete from an $\mathrm{MOO}$ perspective which was not the chosen paradigm in those works anyway. This problem has the advantage that its front (Fig. 3(a)) can be found analytically and the phase transitions derived from it are independent of the number of nodes. We cannot guarantee the same for the variations studied later.

Because we normalized both targets using the clique as a reference, this network is mapped into $(1,1)$ in the $t_{1}-t_{2}$ plane. Any graph will have less edges than a clique, thus the set $\Gamma$ of all connected networks lays below $t_{2}=1$ in the target plane. The lower boundary of $t_{2}$ is achieved by connected networks with the minimum amount of edges possible $(N-1)$. There are
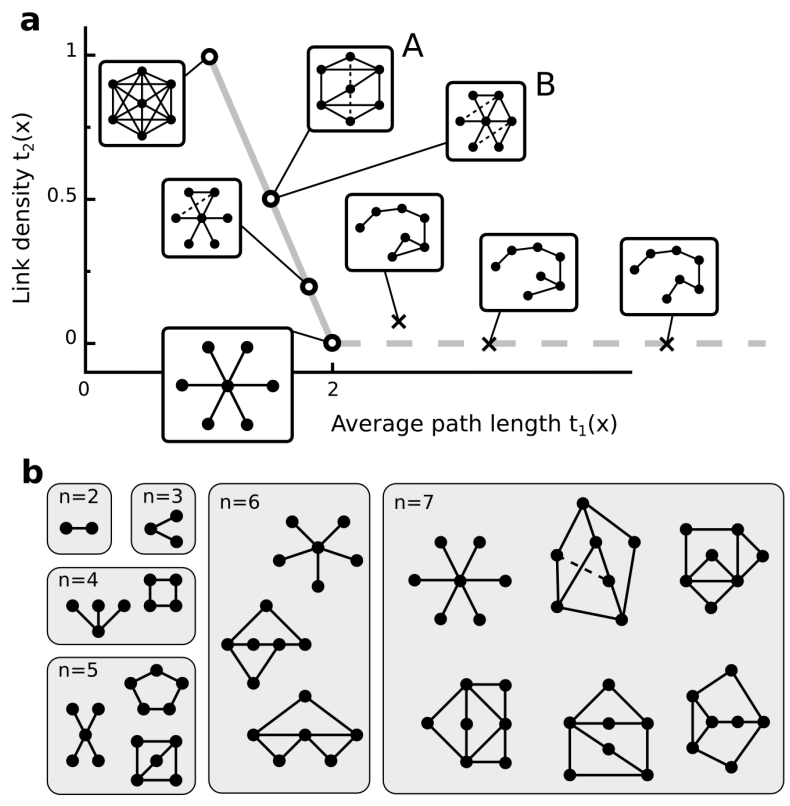

FIG. 3: Pareto front of the fully topological problem. (a) The front (solid gray curve) is a straight line connecting two phases: a star and a clique. The slope of the line $d^{c}=-1$ determines that at $\lambda^{c}=1 / 2$ a first order phase transition takes place. All networks laying on the front are global SOO optima at that critical value. Among them we find networks produced by attaching links to a star and others radically different from the star and from the clique (note the two graphs marked A and B: only one of them can be produced by attaching edges to the star). (b) All core graphs for have been listed for $N \leq 5$. Beyond that, it becomes increasingly difficult to count how many there are or even to tell apart two different ones.

a collection of such graphs, from the star to a linear chain - in between lay all possible trees. All them have $t_{2}=\rho^{t}=1 / N$, which tends to 0 as $N$ goes to infinity. The average path length of these networks varies between that of the $\operatorname{star}(2(N-1) / N \rightarrow 2)$ and the linear chain $\left(\left(N^{2}-1\right) / 3(N-1) \rightarrow+\infty\right)$. These minimally connected graphs lay on a horizontal stretch of the $t_{1}-t_{2}$ plane (dashed line in Fig. 3(a)).

Among these trees (all with the same $t_{2}=1 / N$ ), the star is the one with the lowest average path length, hence it is Pareto optimal. Any other network with a lower $t_{1}$ must have more links than the star, the clique setting the lower $t_{2}$ bound. Thus the Pareto front must lay on a curve connecting the clique and the star - i.e. connecting $(1,1)$ and $(2,0)$ in the $t_{1}-t_{2}$ plane.

We appreciate the following facts: i) The edge density is a function of the number of links alone and it does not depend on the topology of the network. ii) Given a network that is Pareto optimal, we generate new Pareto optimal networks by simply adding new connections. As an instance, the star is Pareto optimal and all its nodes are 1 edge apart from the hub and 2 edges apart from 


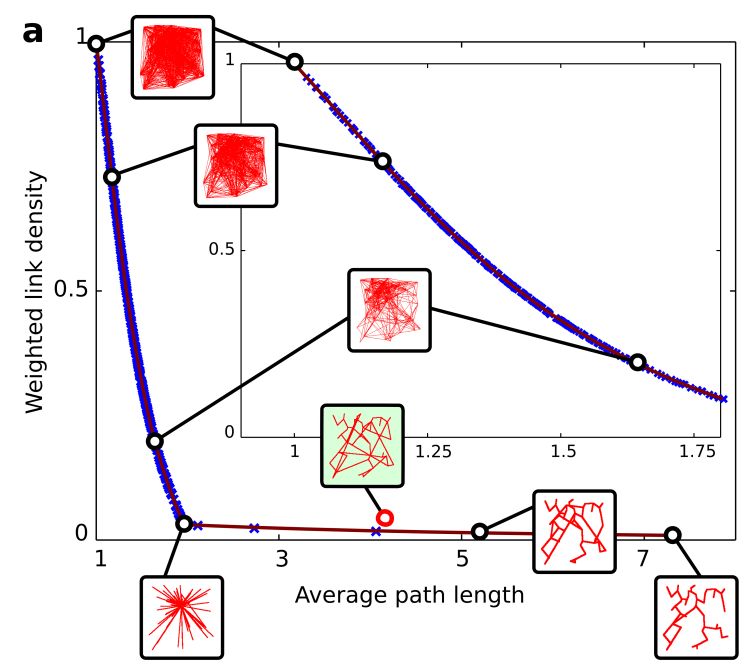

b
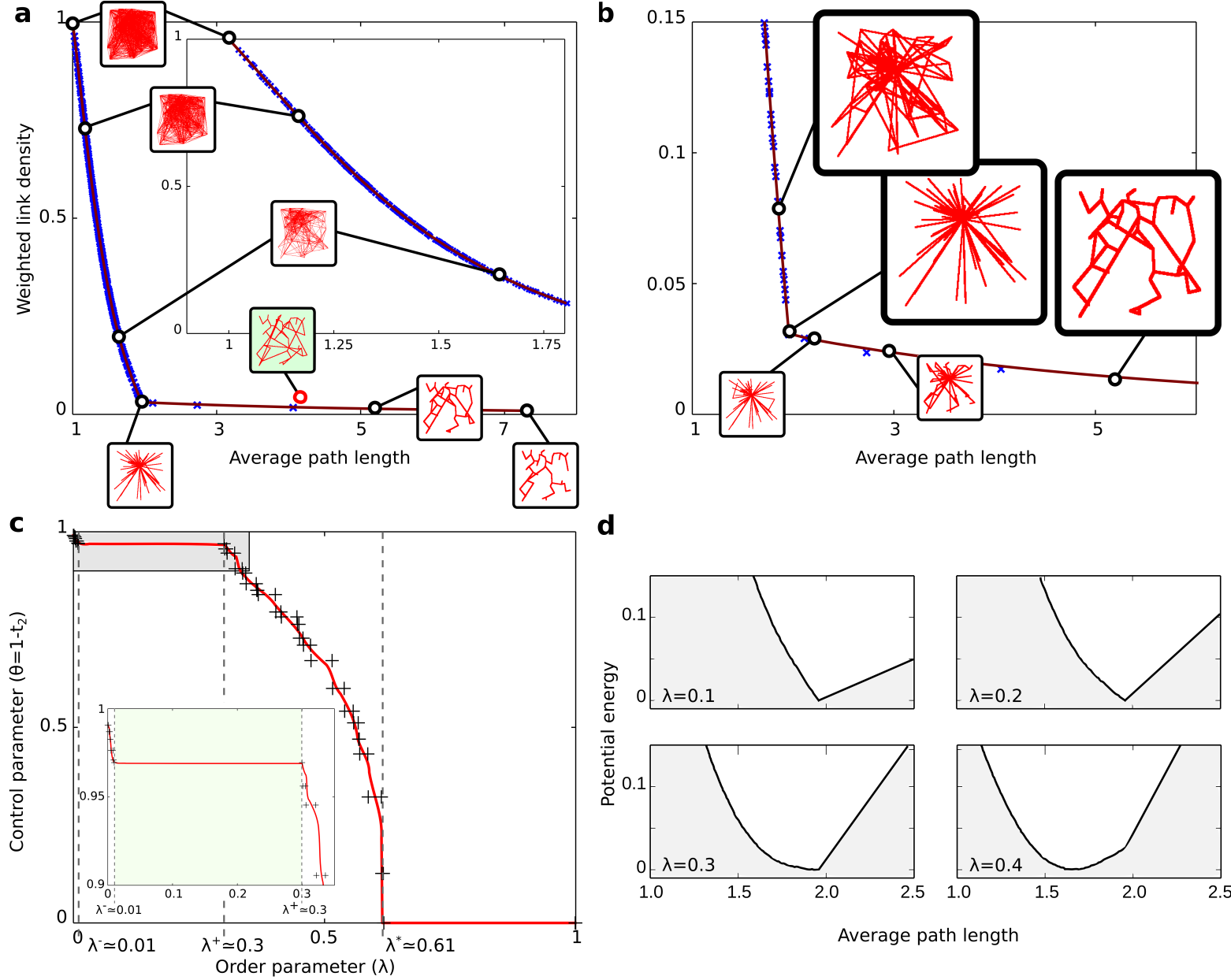

d

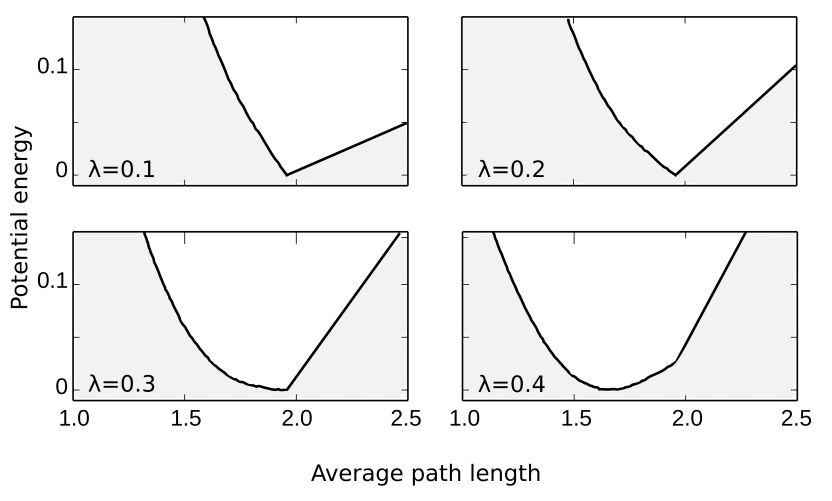

FIG. 4: Partly geometrical problem on nodes scattered over a plane. (a) The front follows the archetype of the topological problem with two roughly perpendicular stretches that trade off between the clique (top-left), the star, and the MST (bottom-right). Incomplete cliques are reached after a second order phase transition because the Pareto front ends abruptly in its top-left (inset). The other extreme of the front ends smoothly. (b) A sharp edge indicates a second order phase transition with the star graph being optimal for a range $\lambda \in\left(\lambda^{-}, \lambda^{+}\right)$. (c) Plotting an order parameter as a function of $\lambda$ reveals both transitions at $\lambda \simeq 0.61$ and at $\lambda^{-} \simeq 0.01$ and $\lambda^{+} \simeq 0.3$ (inset). The star is optimal in the range $\lambda \in\left(\lambda^{-}, \lambda^{+}\right)$, thus any order parameter is constant in that range. (d) The non-analyticity of the Pareto front is inherited by the energetic landscape also as a sharp edge. The $\mathrm{SOO}$ is vividly illustrated thanks to this potential landscape, whose minimum is occupied by one same network for several values of $\lambda$. As lambda changes, the potential well is deformed until the minimum drifts away from the sharp edge.

each other. Then, new edges can only be added that connect directly two non-hub nodes, turning a distance $d^{t}(i, j)=2$ into $d^{t}(i, j)=1$; but not affecting the network in any other respect. Put otherwise, once a network is Pareto optimal any addition of links has got only local effects in its average path length.

Adding new links to the star results in more Pareto optimal networks, the number of which grows combinatorially (that scaling saturates as we approach the clique). Take apart the $N-1$ non-hub nodes of a star: any network that we implement on this subset of nodes (connected or not), and which is subsequently embedded on the original star graph through the hub, is Pareto optimal. It is a sufficient (but not necessary) condition for a network to be Pareto optimal to contain a hub (Fig. $3(\mathrm{a})$ ). The necessary condition for Pareto optimality is that every node is at maximum 2 edges apart from each other.

From any Pareto optimal network (with or without a hub), adding new edges always generates new Pareto optimal graphs. Repeating this operation we always reach a clique, but this process is not reversible: Take the clique and delete connections randomly with the condition that your network remains Pareto optimal after every deletion. No rearrangement of the edges is allowed. Let this process continue until we cannot remove any link without violating the Pareto-optimality condition. This algorithm might yield a star or any other graph from a col- 
lection of irreducible Pareto optimal networks, which we call Pareto core graphs. The star is the core graph with less edges possible. We can only construct these networks as described, since other defining regularities are not apparent - beyond the optimality condition that every node is at most 2 hops away from each other. Some of these graphs are represented in Fig. 3(b) for $N=2, \ldots, 7$. The complexity scales from 1 core graph for $N=2,3$; to two core graphs for $N=4$; to three for $N=5$; to an unknown number for $N \geq 6$. For larger $N$ it also becomes increasingly difficult to determine whether two core graphs are the same, given their invariance under the labeling of the nodes. Note that core graphs are Pareto optimal. They are representative of the staggering complexity contained in the front (which grows combinatorially) and they cannot be trivially composed as a mixture of stars and cliques. Because of this they constitute Pareto and global optima that have not been previously reported.

Even if we cannot list down all Pareto optimal networks, we can find where they live on the $t_{1}-t_{2}$ plane. Adding one edge always modifies $\langle l\rangle^{t}(\gamma)$ by an amount $\Delta\langle l\rangle^{t}=-1 / N(N-1)$, thus $t_{1}$ is decreased. The same operation increases $t_{2}$ by $\Delta \rho^{t}=1 / N(N-1)$. Because $\Delta \rho^{t} / \Delta\langle l\rangle=-1$ does not depend on the number of edges, Pareto optimal graphs thus generated lay on a straight line with slope $d^{c} \equiv \Delta \rho^{t} / \Delta\langle l\rangle$ (Fig. 3(a)). Such a front implies a first order phase transition at $\lambda^{c}=-d^{c} /\left(1-d^{c}\right)=1 / 2$. The clique and the star are found at either phase (correspondingly for $\lambda>\lambda^{c}$ and $\left.\lambda<\lambda^{c}\right)$. Right at the critical value $\lambda^{c}$ any Pareto optimal network is a global optimum. The degenerated complexity at $\lambda=\lambda^{c}$, with so many and structurally different optimal solutions, presents interesting connections with critical phenomena and neutral theory that we explore in future work [40]. The plot of any order parameter as a function of $\lambda$ (not shown) just presents a gap between two constant values.

\section{B. Partly geometrical problem}

Figure 3(a) provides an archetype for the Pareto front that will be repeated (with variations) in the more elaborated MOO problems. Our fronts will present a first, stepped stretch that trades off between the clique (topleft) and some intermediate networks (usually the star); and a second, flat stretch with little variation in the vertical dimension $\left(t_{2}\right)$ and a broad variation in the horizontal axis $\left(t_{1}\right)$. In the previous case, this second stretch (dashed line in Fig. 3(a)) does not belong to the front, but it will in the following problems.

\section{Nodes scattered over a plane}

Figure 4(a) shows the first example of this archetype. A very stepped stretch of the front trades off between the clique and the star just as before. However, this is a convex curve now, which we discuss below. The second archetypal stretch of the front trades off between the star and the MST, and is mapped onto an almost horizontal curve in the $t_{1}-t_{2}$ plane with a slight convexity. This Pareto front ends up smoothly in its bottom-right extreme, so we dismiss any phenomenon associated to it. Because the whole front is convex first order phase transitions are ruled out.

The first, stepped stretch of the front (Fig. 4(a), inset) presents a feature that appears in most subsequent cases. This stretch is a convex curve that ends abruptly (with the notion of abruptness introduced before). This indicates that a second order phase transition takes place. This transition trades off between the clique (persistent global optimum for $\lambda>\lambda^{*} \simeq 0.61$ ) and dense but incomplete graphs reached as we move below $\lambda^{*}$. Because the clique is optimal for $\lambda \geq \lambda^{*}$, anything that we measure on this global optimum stays constant as a function of $\lambda$ until $\lambda<\lambda^{*}$, for which our wandering over the Pareto front yields a changing global optimum as $\lambda$ decreases. Then, any measurement performed on the SOO optimum will vary steadily with a derivative (with respect to $\lambda$ ) different from 0 . This discontinuity in the derivative indicates that a second order phase transition takes place (Fig. $4(\mathrm{c}))$.

A sharp edge in the front (Fig. 4(b)) indicates yet another second order phase transition similar to that described in Fig. 2(b): The slope of the front is well defined as we tend towards the sharp edge from the left (yielding a slope $d^{+}$such that $\left.\lambda^{+}=-d^{+} /\left(1-d^{+}\right) \simeq 0.3\right)$, and as we tend to the sharp edge from the right (now with $d^{-}$ such that $\left.\lambda^{-}=0.01\right)$. For any $\lambda \in\left(\lambda^{-}, \lambda^{+}\right)$, SOOs are well defined through equation (4); but there are not any points of the front with a slope $-\lambda /(1-\lambda)=d \in\left(d^{+}, d^{-}\right)$ where to locate the optimum. Instead, the same one network laying precisely at the sharp edge is consistently optimal for this range of $\lambda$. Anything that we measure about this optimum will remain as a function of $\lambda$ in $\left(\lambda^{-}, \lambda^{+}\right)$, but samplings of the front run smoothly below $\lambda^{-}$and above $\lambda^{+}$, with well defined derivatives for any order parameter as a function of $\lambda$. Hence, two discontinuities are evident in this derivative (Fig. 4(c), inset).

Qualitatively, browsing the front through $\lambda$ is a continuous transition from the clique (which is a global optima for a wide range of $\lambda$ ), through the star graph (also a persistent optimum for a continuum of $\lambda$ ), to the MST. The weighted density of edges $\left(t_{1}=\rho^{g}\right)$ penalizes large connections first, which are dropped as we leave the clique. But enough of them survive among Pareto optimal graphs so that the star can be reached continuously, without needing a drastic rewiring that would leave an imprint in the order parameters. Note that these few long edges survive because they enable a low average path length $\left(t_{2}=\langle l\rangle^{t}\right)$, which is still measured as the number of hops between nodes. Finally, to rearrange the star into the MST, the surviving long edges are replaced by winding branches that extend visiting many nodes on their 
a
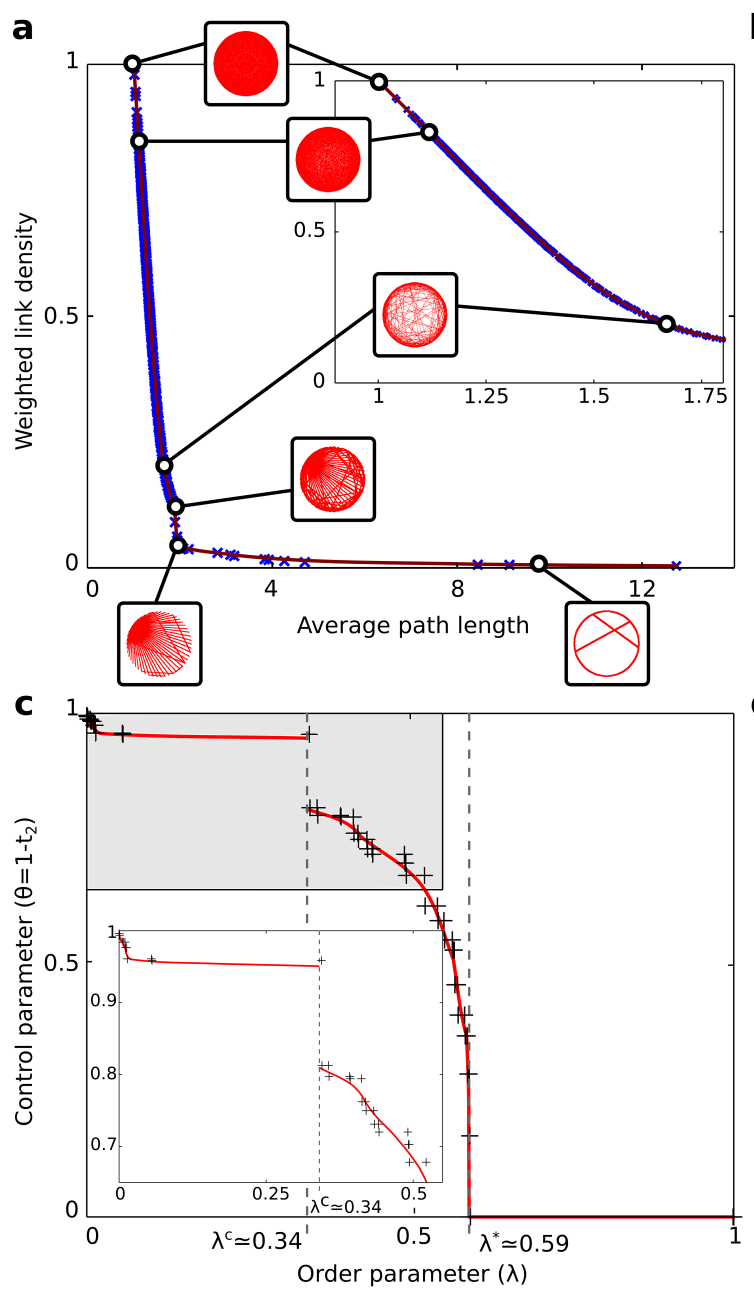

b

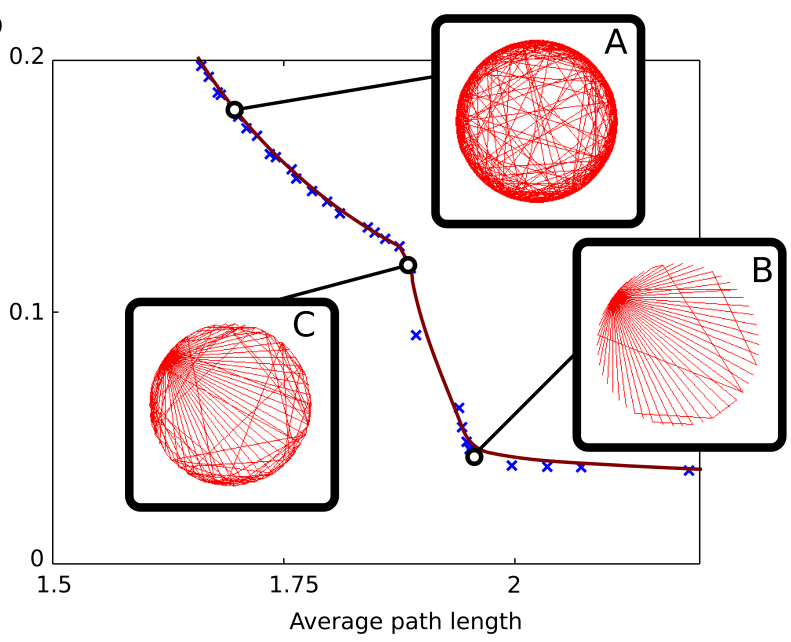

d
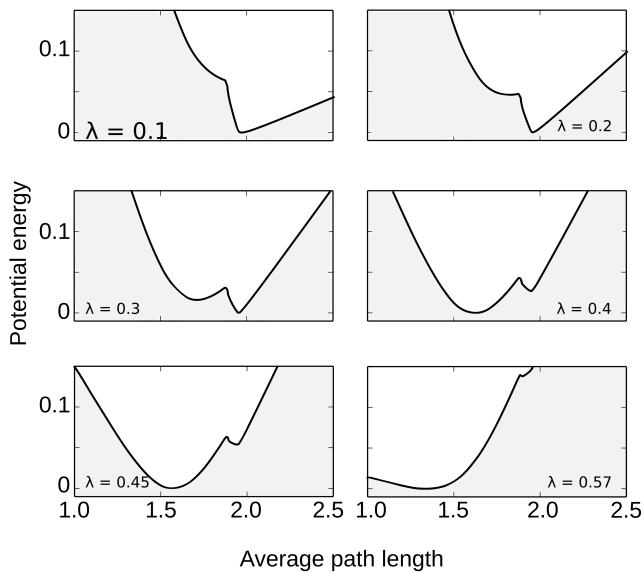

FIG. 5: Partly geometrical problem on a circle. (a) Again, the front follows the archetype of the topological problem with two roughly perpendicular stretches that trade off between the clique (top-left), star networks, and the MST. Around the clique it is observed the same phase transition as before. In the cavity it is solved a complex rearrangement. Networks that drop their larger connections first (A) must morph into a star (B), which requires some of these far-reaching edges. Therefore, some Pareto optimal networks are produced that never get to be SOO optima (C), yielding a first order transition. (c) Order parameters as a function of $\lambda$ reveal the first $\left(\lambda^{c} \simeq 0.34\right.$, magnified in the inset) and second $\left(\lambda^{*} \simeq 0.59\right)$ order transitions. (d) The landscape potential unveils the mechanisms for local equilibrium and hysteresis associated to first order transitions. At low levels of the control parameter $(\lambda \sim 0.1)$ only one minimum exists in the global energy $\Omega$. A pocket becomes locally stable for $\lambda \sim 0.2$. This grows for larger $\lambda$, until it becomes the global extreme of the energetic landscape $(\lambda \sim 0.4)$. Optimizing our networks through numerical algorithms can get us stuck in local minimums, so to transit from one potential well to the other we need to increase our control parameter until one of the wells get destabilized $(\lambda \sim 0.57)$. Repeating the operation with decreasing $\lambda$ can get us stuck in the other well, thus engaging in a hysteresis loop. Some Pareto optimal networks inside the cavity are reached at these metastable states.

way. Alternative strategies, like hybrid MSTs that incorporate non-essential shortcuts between far-apart nodes, fall off the Pareto front (note the sub-optimal graph highlighted in Fig. 4(a)).

It is very useful to consider the potential landscape introduced by equation (4) to stretch our intuition. For a fixed value of $\lambda$ we compute the energy $\Omega(\gamma, \lambda)$ for every Pareto optimal network. The result is a lower energy boundary (Fig. 4(d)). Not Pareto-optimal networks must present yet higher energies. The SOO solution becomes now very intuitive since the global optimum lays at the minimum of $\Omega(\gamma, \lambda)$, which has got a vivid graphic representation. But this potential landscape changes as a function of $\lambda$, and consequently its minimum. The sharp edge of the Pareto front is inherited by the potential landscape, which presents a persistent minimum for a range of the control parameter. 


\section{Nodes spaced over a circle}

The front of this problem (Fig. 5(a)-(b)) again follows the archetype: i) a stepped stretch to the left that trades-off between the clique and dense (yet incomplete) graphs and ii) a flatter stretch that encompasses graphs with roughly the same edge density but a wide variation along the average path length dimension. This second stretch extends to large values of $t_{1}$ : a region populated by minimal, circlelike networks with little long-range connections. It seems a convex stretch that ends smoothly, so nothing remarkable happens there. Again, the stepped stretch of the front is convex (Fig. 5(a), inset) and ends abruptly revealing a second order phase transition. It is similar to the one encountered before by the clique and happens at a similar value $\lambda \simeq 0.59$ (Fig. 5 (c)).

The notable feature that this front introduces is a concavity at the junction between the two archetypal stretches of the front (Fig. 4(b)). The cavity lays at the confluence between the three relevant network topologies: incomplete cliques, the star, and encircled nets (the MST of the problem). As in the previous case, longer edges are dropped first. The scattered nodes managed to retain enough long-range links in that example, enabling a continuous transition through the star. This is not possible now, and the symmetry of the circle might be crucial therefore. Earlier, the distribution of lengths were varied, while now all long-range edges are the same: the moment one is dropped, the others follow. As we leave the clique, we converge quickly to encircled graphs with little long range connections; and these lay inside a cavity of the front (Fig. 4(b)). To reconstruct a star (which remains Pareto optimal due to its low average path length) a drastic rewiring in unavoidable. This prompts a first order phase transition $\left(\lambda^{c} \simeq 0.34\right)$ whose imprint is, indeed, that cavity. That transition is reflected in any order parameter $\theta$ that we plot as a function of $\lambda$ (Fig. 4(c), inset).

We can resort again to a potential landscape to visualize this transition. Plotting $\Omega(\gamma, \lambda)$ for all Pareto optimal networks we obtain the lower energy boundaries portrayed in Fig. 5(d). This landscape changes as $\lambda$ varies, producing two potential wells associated to local minimums. At $\lambda=\lambda^{c}$, both minimums present the same energy, thus both phases coexist. Moving away from the transition point, one of the wells is unstabilized. Note that moving $\lambda$ back and forth could get us temporarily trapped in metastable states (the most energetic local minimum) and hysteresis loops would be observed.

\section{Fully geometric problem}

Introducing geometry in both target functions has the effect of smoothing the Pareto front, removing first order phase transitions. Some relevant second order transitions disappear. Others persist, but only at the extremes of the front. The picture becomes closer to a soft trading-off between the clique and the MST.

1. Nodes scattered over a plane

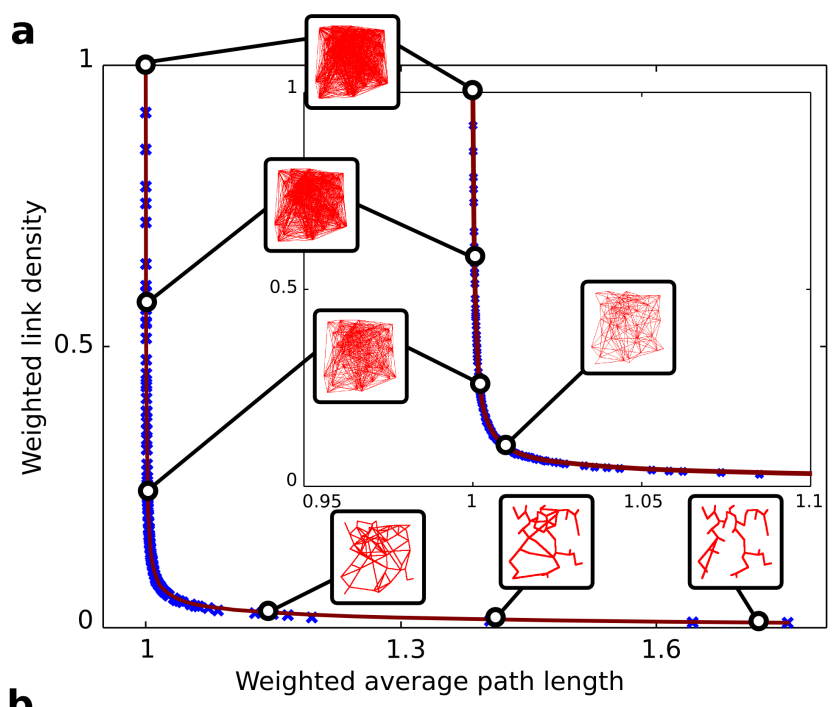

b

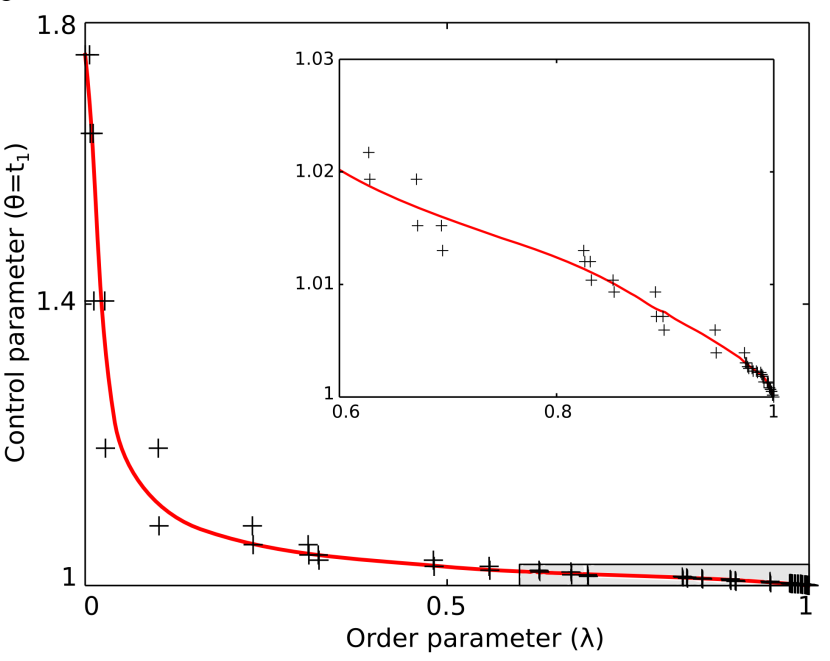

FIG. 6: Fully geometrical problem for nodes scattered over a plane. (a) The front has no accidents. It is completely convex and spans all possible slopes so that each $\lambda \in(0,1)$ poses an $\mathrm{SOO}$ with a different solution. As we roll over the front, the clique gently leads to less connected networks, towards the MST. (b) The absence of phase transitions renders smooth plots of any order parameters.

This problem presents a quite uninteresting front (Fig. 6(a)) without phase transitions. The front spans all possible slopes $d \in(-\infty, 0)$, so that SOOs with different solutions can be posed for each $\lambda \in(0,1)$. Any order parameter renders a continuous plot (Fig. 6(b)) even when zooming in to tiny details (inset). The first derivative of the order parameters also behaves properly.

All phase transitions from previous cases have vanished. Besides the geometric disposition of the nodes 
(which has some obvious influence over what transitions are present), it is notable that choices of optimization targets exist for which previously existing transitions disappear. The fact that, given a same set of networks $\gamma \in \Gamma$, a choice of targets erases previous transitions implies that a gentle evolution between radically different topologies (clique and MST) can happen, despite the drastic modifications that we might envision necessary a priori, and despite the phase transitions that do take place on the same graphs for other choices of targets. This stresses the role of target functions to frame phase transitions properly.

\section{Nodes spaced over a circle}

Again, introducing geometry in both targets smooths the Pareto front (Fig. 7(a)). The first order transition found in the circle before has disappeared. There is no cavity now and the tradeoff between clique and circle happens gradually as we roll over the front. That previous transition took place because the gradual drift from clique to circle was interrupted by the presence of the Pareto optimal star, which kept the average path length low because it was measured as the number of hops between nodes. But now geometry also enters through $t_{1}=\langle l\rangle^{g}(\gamma)$ and using only two links to get from one node to another is still costly if these are far reaching connections. It is more economic now to circle around even if that implies visiting many more nodes. Thus the star is retracted from the Pareto front and the transition from the clique to the MST proceeds smoothly.

The bottom stretch of the front seems convex but abruptly terminated, suggesting a second order transition at $\lambda_{1}^{*} \simeq 0.28$ that trades off between the MST (an almost complete circle, which is persistently optimal for $\lambda \leq \lambda_{1}^{*}$ ) and other, more connected graphs. The characteristic plot of a second order transition is noted in any order parameter (Fig. 7(b)). At the other end of the front we find the usual transition associated to the clique, which did not disappear but has been moved to $\lambda_{2}^{*} \simeq 0.98$. The same characteristic order parameter plot can be appreciated (Fig. 7(b), inset). For $\lambda \in\left(\lambda_{1}^{*}, \lambda_{2}^{*}\right)$ any order parameter is a smooth function of $\lambda$.

\section{CONCLUSIONS}

In this paper we solve three MOOs defined on complex networks. These problems allow us to explore interesting aspects of Pareto optimality. Following recent contributions in biology [41, 42, our work is an exploration of a morphospace. A first approach to such spaces is to list all possible morphologies for a system and locate them quantitatively with respect to some relevant aspects here, complex networks are characterized in an averagepath-length vs. edge-density two-dimensional space. We propose that a natural selection process based on Pareto
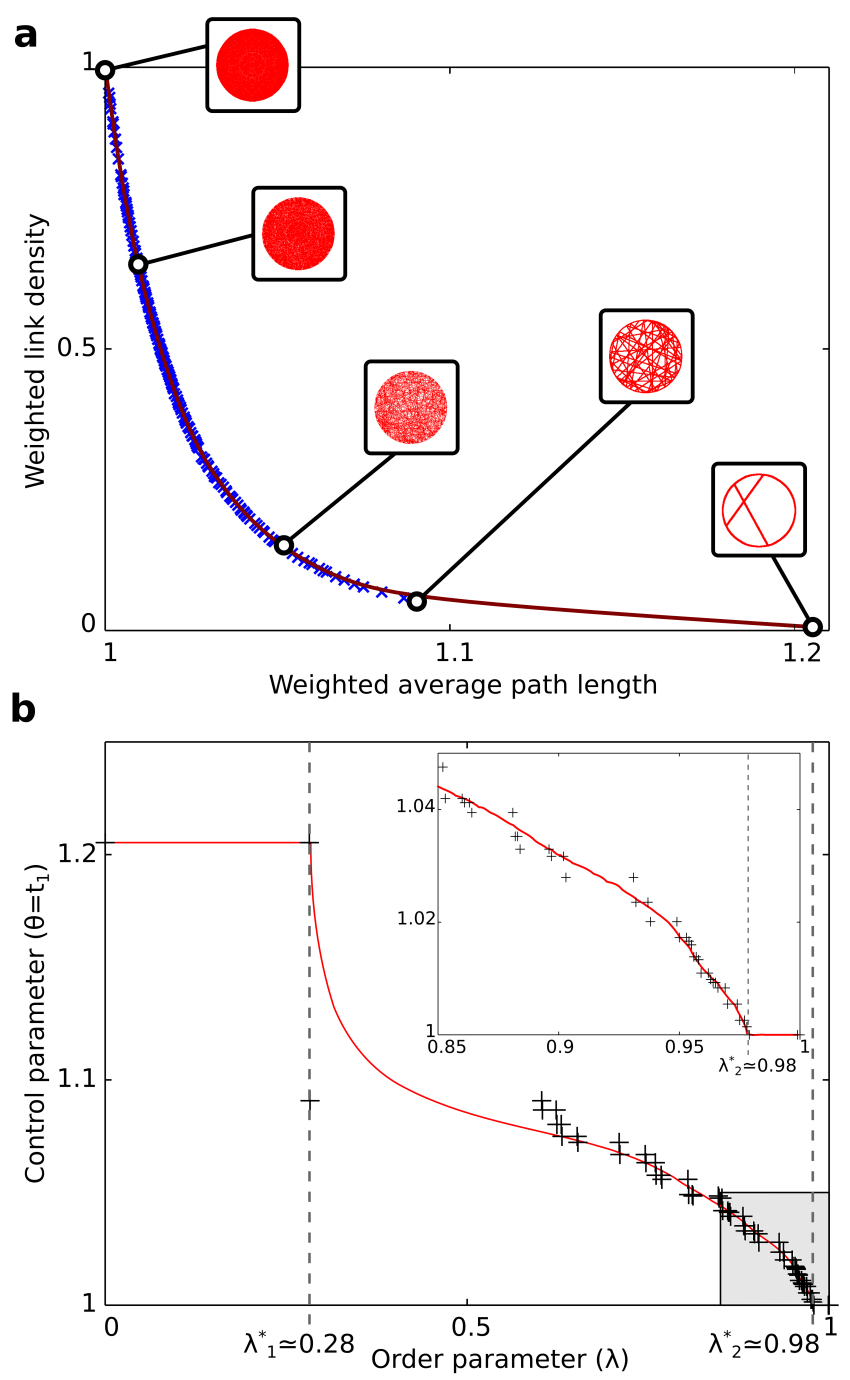

FIG. 7: Fully geometrical problem on a circle. (a) The front presents a smooth transition between the clique and the open circle, with no relevant feats except in the extremes of the front. These end up abruptly, as in second order phase transitions. (b) Plotting any order parameters reveals these phase transitions at $\lambda_{1}^{*} \simeq 0.28$ and $\lambda_{2}^{*} \simeq 0.98$ (inset).

optimality shall constrain further such a morphospace and we study the effects of these conflictive restrictions. In doing so, we follow recent works 43 46 that illustrate how Pareto optimality reduces the effective dimensionality of certain complex systems.

Alternatively, a recent theoretical framework brings together statistical mechanics and MOO [35], thus enriching the analysis of Pareto optimal designs. This framework reveals universal features of Pareto optimal systems that correspond to phase transitions or critical phenomena.

First order phase transitions indicate that a system must undergo important structural changes despite little variation of some control parameter. In thermodynam- 
ics, this implies great investments of energy in reshaping matter, e.g., as it transits from solid to fluid. Similar demands might be requested of complex Pareto optimal systems, specially if the parameters controlling the conditions for optimality may change over time. This underscores the importance of gathering knowledge about the Pareto front before implementing solutions to any optimization problem.

Second order phase transitions can also be very informative about the nature of a system. They indicate that some solution is stable for a large range of the control parameters, thus making it more likely if evolution or design has taken place under many different scenarios. On the other hand, if such stable solutions would not show up in an evolutionary setup, we would have strong evidence that a large set of possible circumstances do not occur naturally.

We have found a variety of first and second order transitions. These depend very much on the precise mathematical expression of the optimization targets (see, for example, how transitions disappear as we change the measure of average path length). Hence, looking at the problem from an alternative perspective, the presence or absence of expected phase transitions in real systems could be informative about the nature of the optimization pressures that these systems might be subjected to.

Finally, the fully topological problem presents a quite singular case: a first order phase transition takes place between the star graph (for $\lambda<\lambda^{c}$ ) and the clique $\left(\lambda>\lambda^{c}\right)$, while all other Pareto optimal networks are also global optima at $\lambda=\lambda^{c}$. This would suggest that cliques and star graphs should happen overwhelmingly more often than any other topology when geometry is not relevant, unless every optimal network had evolved under the unlikely condition $\lambda=\lambda^{c}$. This is notably at odds with the reality and a possible solution (with connections to critical phenomena) will be explored elsewhere [40].

\section{Acknowledgments}

We thank the members of the CSL for useful discussions. This work was supported by grants from the Fundación Botín, the European Research Council (ERC Advanced Grant), and by the Santa Fe Institute.

\section{Appendix A: Analytic and numeric approaches for MOO solving}

We relied on genetic algorithms to locate the different Pareto fronts. The fully topological case can be solved analytically, but the same genetic algorithm was used to check for good convergence showing very good results. In this appendix we explain in detail the genetic algorithm. Following that explanation, a few disclaimers are in order about the numerical nature of the solutions found i.e. about the fact that convergence to the Pareto front cannot be guaranteed and the smoothing necessary to render a continuous approximation to the front.

\section{A multiobjective genetic algorithm}

MOO relays on the concept of Pareto dominance. Given two networks $\gamma_{i}, \gamma_{j} \in \Gamma$, both mapped into $\mathbb{R}^{2}$ through $t_{1}\left(\gamma_{i / j}\right)$ and $t_{2}\left(\gamma_{i / j}\right)$, we say that $\gamma_{i}$ dominates $\gamma_{j}$ (and note it $\gamma_{i} \prec \gamma_{j}$ ) if $\gamma_{i}$ is not worst than $\gamma_{j}$ regarding any target and it is better than $\gamma_{j}$ with respect to at least one target. We can visualize this: Since we deal with minimizations in the $t_{1}-t_{2}$ plane, network $\gamma_{i}$ has got a set of axes associated with their origin at $\left(t_{1}\left(\gamma_{i}\right), t_{2}\left(\gamma_{i}\right)\right)$ and every network $\gamma_{j}$ laying on the first quadrant of these axes is Pareto dominated by $\gamma_{i}$.

Following the literature on multiobjective genetic algorithms [47 49] we computed a dominance score: We took the set $D_{j} \equiv\left\{\gamma_{i} \mid \gamma_{i} \prec \gamma_{j}\right\}$ of solutions from within a given population (an arbitrary subset of $\Gamma$ ) that dominate $\gamma_{j}$. The size of this set $\left(d_{j}=\left\|D_{j}\right\|\right.$, dubbed the dominance score), indicates how fit $\gamma_{j}$ is in terms of Pareto optimality. We proceeded then to minimize this score. We departed from an initial population of $N_{P}$ networks (either random or designed, see below), selected $N_{P} / 2$ of the population based on the dominance score, chose random pairs among the selected networks to produce $N_{P} / 2$ new networks, and applied random mutations to all but a subset of elite networks. We iterated this scheme a fixed number of generations.

Mutations consisted in random appending or deleting edges or totally swapping the connections of two arbitrary nodes. For crossover, from each of the two mating nets we assigned each node and its connections randomly to each of the offspring graphs checking that the same node and connection was not assigned twice to the same child. After crossover or edge deletion we checked that all networks remained connected all the time. We completed one missing link whenever connectedness failed and then checked for connectedness again.

All we care about for the current research is good convergence towards the front. This justifies our using of crossover: this is a very good evolutionary operator, though unrealistic if we wanted to study some features of nature. For example, such an operator would not be adequate to study species that do not reproduce sexually. Studying Pareto optimality under constrained conditions - e.g. without crossover - also renders a set of non-dominated solutions. These might converge to the Pareto front or not, and they might be subjected to geometrical constraints in $t_{1}-t_{2}$ that are similar to those studied in 35] and in this paper for Pareto optimal networks. Such constrained evolutionary schemes pose interesting research questions, but here we are concerned with Pareto optimal solutions. This justifies the crossover and a clever initialization of the algorithm. It might be difficult to converge towards some solutions that are highly non-trivial - e.g. the MST. We know, though, that this 
solution belongs to the front of all physically grounded problems. If an algorithm would fail in finding this solution, this could hinder convergence towards an interesting (though challenging) region of the Pareto front. Once again, because we are concerned with Pareto optimal solutions and would like to attain the closest convergence possible, it is also fully justified to seed the initial population with a few designed solutions. We did so by introducing since the very beginning cliques, stargraphs, MSTs, and circle networks (these two are equivalent for the circle) with very slight mutations. We produced $N_{P} / 4$ of each such major topologies at the beginning. The crossover and mutation operators ensure fast exploration of hybrid topologies.

As noted above, we know that some of these networks are Pareto optimal: the clique is always so, and the MST is Pareto optimal in all physically grounded problems. However, and since we started with little variations upon these graphs, the algorithm did not always reach these solutions - but it surely explored the region nearby. There might be other interesting regions of the front that might not have been fully explored and that are impossible to seed without foreknowledge. Although we are concerned with Pareto optimal solutions alone, our methods are numerical at the end and convergence of multiobjective genetic algorithms to the Pareto front cannot be guaranteed. We decided to report on the results of the simulations with as little reinterpretation and further speculation as possible. Notwithstanding, the overall details of the Pareto front seem to be recovered and the theory posed in [35] is properly illustrated.

As for the implementation of the algorithm, we used a population of $N_{P}=3000$ connected networks with $N=50$ nodes - with the initial population seeded as indicated above. The population was evolved during $T=10000$ generations in every case. Mutation happened with a probability $p_{\mu}=0.001$ of appending an extra link to each network, the same probability of deleting an existing edge, and the same probability of swapping the ends of each existing connection. The top $N_{e}=50$ networks of the population where considered elite and were spared any mutation. As the algorithm proceeds, many networks reach a dominance score of 0 even if they are not Pareto optimal. Unluckily, this score is the best indicator of Pareto optimality available (not only in the current implementation, but generally). This results in elite members of the population not being objectively better than non-elite members - in terms of Pareto optimality. Because the algorithm sorted the population consistently from one generation to the next, what members of the population are considered elite is largely a matter of antiquity: early members that reach low dominance score and are not overthrown are likely to be preserved during the whole simulation. We repeated 4 times the simulations with scattered nodes to check that the relevant features obtained were not artifacts of some lucky distributions of the nodes.

\section{Smoothing of the front and order parameters}

The only speculation that we allowed ourselves is in choosing a relevant scale for analysis, which led to a smoothing of the Pareto front. As noted in Sec. III, we deal with a discrete set of networks whose front is necessarily discrete as well. Accordingly, every shift in global optima is a first order phase transition at some scale and global optima remain so for a continuous range of $\lambda$, as in second order phase transitions. This does not further our understanding of the problem as much as a coarse-grained analysis that renders noteworthy phase transitions. Since the genetic algorithm only produces a finite set of (ideally) Pareto optimal solutions, we applied a Bezier smoothing to their plot on the $t_{1}-t_{2}$ plane. We took care that the smoothing did not introduce alien concavities. Because Bezier curves cannot present sharp edges (thus ruling out second order phase transitions), when a sharp edge seemed the best description of the front (Sec. IIIB partly geometrical problem on nodes scattered over a plane), we decided to split the front in its two salient branches and apply two independent smoothing processes that allowed us to recover the transition in great detail.

To locate global optima, we calculated $\Omega\left(x_{\Pi}, \lambda\right)$ for the optimal solutions produced by the genetic algorithm, and for a large sample of points from the Bezier curves introduced in the previous paragraph. We registered the global optimal for different values of $\lambda$. One of the problems pointed at earlier is that, because of the discreteness of the front, global optima are so for several values of $\lambda$. This would cause that the plots of order parameters look tiered. For a better illustration of the results, whenever order parameters are plotted we indicate only the first and last values of $\lambda$ for which each global optima are indeed optima (black crosses in all order parameter plots). The smoothing allows a finner grained sampling so that this is not an issue: the corresponding order parameters (red curves in all order parameter plots) look continuous always.

Following [35, anything well behaved that we measure upon global optima are accepted as order parameters. By well behaved we imply that order parameters should not introduce alien divergences into the problem, and that solutions laying at different points over the front should score differently in this parameter. This way we ensure that any feature stemming from the optimization problem does not go unreported and that we do not introduce phase-transition-like behaviors that originated, e.g., on some function diverging to infinity for reasons of its own. Taking these guidelines into account, the target functions themselves are always good order parameters. We use these $\left(\theta=t_{1}\right.$ in Sec. III C), or trivial transformations of them $\left(\theta=1-t_{2}\right.$ in Sec. III B). More drastic transformations such as $1 /\left(2-t_{1}\right)$ would be banned: note that this function diverges for $t_{1}=2$ even if this is a perfectly regular point of the front for all problems. 
[1] R. Dawkins, Climbing mount improbable (WW Norton \& Company, 1997).

[2] H. M. Ozaktas, Paradigms of connectivity for computer circuits and networks. Opt. Eng. 31, 1536 (1992).

[3] W. K. Chen, The VLSI Handbook (CRC Press, Boca Raton, Fl, 1999).

[4] D. S. Bassett, D. L. Greenfield, A. Meyer-Lindenberg, D. R. Weinberger, S. W. Moore, and E. T. Bullmore, Efficient Physical Embedding of Topologically Complex Information Processing Networks in Brains and Computer Circuits. PLoS Comput. Biol 6(4), e1000748 (2010).

[5] D. J. Watts and S. H. Strogatz, Collective dynamics of 'small-world' networks. Nature 393, 440 (1998).

[6] L. A. N. Amaral, A. Scala, M. Barthélémy, and H. E. Stanley, Classes of small-world networks. P. Nat. A. Sci. 97(21), 11149 (2000).

[7] M. Barthélemy, Spatial networks. Phys. Rep. 499(1), 1 (2011).

[8] K. J. Kansky, PhD thesis, University of Chicago, 1963. Structure of transportation networks: relationships between network geometry and regional characteristics, $\mathrm{PhD}$ Thesis,

[9] F. R. Pitts, A graph theoretic approach to historical geography. Prof. Geogr. 17(5), 15 (1965).

[10] P. Sen, S. Dasgupta, A. Chatterjee, P. A. Sreeram, G. Mukherjee, and S. S. Manna, Small-world properties of the Indian railway network. Phys. Rev. E 67(3), 036106 (2003).

[11] M. T. Gastner and M. E. J. Newman, Optimal design of spatial distribution networks. Phys. Rev. E 74, 016117 (2006).

[12] R. Carvalho, L. Buzna, F. Bono, E. Gutiérrez, W. Just, and D. Arrowsmith, Robustness of trans-European gas networks Phys. Rev. E 80, 016106 (2009).

[13] J. Clune, J. B. Mouret, and H. Lipson, The evolutionary origins of modularity. Proc. R. Soc. B, 280(1755), 20122863 (2013).

[14] H. Mengistu, J. Huizinga, J. B. Mouret, and J. Clune, The evolutionary origins of hierarchy. arXiv preprint arXiv:1505.06353 (2015).

[15] C. D. Murray, The physiological principle of minimum work. I. The vascular system and the cost of blood volume. Proc. Nat. Acad. Sci. 12(3), 207 (1926).

[16] G. B. West, J. H. Brown, and B. J. Enquist, A General Model for the Origin of Allometric Scaling Laws in Biology. Science 276, 122 (1997).

[17] G. B. West, J. H. Brown, and B. J. Enquist, A general model for the structure and allometry of plant vascular systems. Nature 400, 664 (1999).

[18] J. R. Banavar, A. Maritan, and A. Rinaldo, Size and form in efficient transportation networks. Nature 399(6732), 130 (1999).

[19] V. V. Gafiychuk and I. A. Lubashevsky, On the Principles of the Vascular Network Branching. J. Theor. Biol. 212, 1 (2001).

[20] H. Cuntz, A. Borst, and I. Segev, Optimization principles of dendritic structure. Theor. Biol. Med. Model. 4, 21 (2007).

[21] A. Pérez-Escudero and G. G. de Polavieja, Optimally wired subnetwork determines neuroanatomy of Caenorhabditis elegans. P. Nat. A. Sci. 104(43), 17180
(2007).

[22] A. Hasenstaub, S. Otte, E. Callaway, and T. J. Sejnowski, Metabolic cost as a unifying principle governing neuronal biophysics. P. Nat. A. Sci. $107(27), 12329$ (2010).

[23] H. Cuntz, F. Forstner, A. Borst, and M. Häusser, One Rule to Grow Them All: A General Theory of Neuronal Branching and Its Practical Application. PLoS Comput. Biol. 6(8), e1000877 (2010).

[24] V. J. Wedeen, D. L. Rosene, R. Wang, G. Dai, F. Mortazavi, P. Hagmann, J. H. Kaas, and W.-Y. I. Tseng, The Geometric Structure of the Brain Fiber Pathways. Science 335, 1628 (2012).

[25] R. Ferrer i Cancho and R. Solé, Optimization in complex networks. In Statistical Mechanics of Complex Networks 625, 114 (2003).

[26] M. E. J. Newman, Networks: an introduction (Oxford University Press, 2010), Chap. 14.

[27] V. Colizza, J. R. Banavar, A. Maritan, and A. Rinaldo, Network Structures from Selection Principles. Phys. Rev. Lett. 92(19), 198701 (2004).

[28] R. Ferrer i Cancho and R. Solé, Least effort and the origins of scaling in human language. P. Nat. A. Sci. 100(3), 788 (2003).

[29] M. Prokopenko, N. Ay, O. Obst, and D. Polani, Phase transitions in least-effort communications. J. Stat. Mech. 11, P11025 (2010).

[30] C. Salge, N. Ay, D. Polani, and M. Prokopenko, Zipf's Law: Balancing Signal Usage Cost and Communication Efficiency. SFI working paper: 13-10-033 (2013).

[31] N. Mathias and V. Gopal, Small-worlds: how and why. Phys. Rev. E 63, 021117 (2001).

[32] C. M. Fonseca and P. J. Fleming, An Overview of Evolutionary Algorithms in Multiobjective Optimization. Evol. Comput. 3, 1 (1995).

[33] C. A. Coello, Evolutionary Multi-Objective Optimization: A Historical View of the Field. IEEE Comput. Intell. M. 1(1), 28 (2006).

[34] P. Schuster, Optimization of multiple criteria. Complexity 18, 5 (2012).

[35] L. F. Seoane and R. Solé, A multiobjective optimization approach to statistical mechanics. http://arxiv.org/abs/1310.6372

[36] J. W. Gibbs, A Method of Geometrical Representation of the Thermodynamic Properties of Substances by Means of Surfaces. Trans. Conn. Acad. 2, 382 (1873).

[37] J. C. Maxwell, Theory of Heat (Longmans, Green, and Co., 1904), p. 195.

[38] R. Louf, P. Jensen, and M. Barthélemy, Emergence of hierarchy in cost-driven growth of spatial networks. $P$. Nat. A. Sci. 110(22), 8824 (2013).

[39] J. Goñi, A. Avena-Koenigsberger, N. V. de Mendizabal, M. van den Heuvel, R. Betzel, and O. Sporns, Exploring the morphospace of communication efficiency in complex networks. PLoS ONE 8, e58070 (2013).

[40] L. F. Seoane and R. Solé, Systems poised to criticality through Pareto selective forces. In preparation (2015).

[41] L. da Fontoura Costa, K. Zawadzki, M. Miazaki, M. P. Viana, and S. N. Taraskin, Unveiling the neuromorphological space. Frontiers Comput. Neurosci. 4, 150 (2010).

[42] A. Avena-Koenigsberger, J. Goñi, R. Solé, and O. Sporns, Network morphospace. J. R. Soc. Interface 
12(103), 20140881 (2015).

[43] O. Shoval, H. Sheftel, G. Shinar, Y. Hart, O. Ramote, A. Mayo, E. Dekel, K. Kavanagh, and U. Alon, Evolutionary tradeoffs, Pareto optimality, and the geometry of phenotype space. Science 336(6085), 1157 (2012).

[44] R. Schuetz, N. Zamboni, M. Zampieri, M. Heinemann, and U. Sauer, Multidimensional Optimality of Microbial Metabolism. Science 336(6081), 601 (2012).

[45] H. Sheftel, O. Shoval, A. Mayo, and U. Alon, The geometry of the Pareto front in biological phenotype space. Ecol. Evol. 3(6), 1471 (2013).

[46] P. Szekely, H. Sheftel, A. Mayo, and U. Alon, Evolutionary tradeoffs between economy and effectiveness in bio- logical homeostasis systems. PLoS Comput. Biol. 9(8), e1003163 (2013).

[47] F. M. Dittes, Optimization on Rugged Landscapes: A New General Purpose Monte Carlo Approach. Phys. Rev. Lett. 76(25), 4651 (1996).

[48] E. Zitzler, PhD thesis, Swiss Federal Institute of Technology Zurich, 1999. Evolutionary Algorithms for Multiobjective Optimization: Methods and Applications,

[49] A. Konak, D. W. Coit, and A. E. Smith, Multi-objective optimization using genetic algorithms: A tutorial. Reliab. Eng. Syst. Safe. 91(9), 992 (2006). 